\title{
Update on phenomenological extraction of the proton tensor charge
}

Marco Radici*

INFN Sezione di Pavia, via Bassi 6, I-27100 Pavia, Italy

E-mail: marco.radici@pv.infn.it

We discuss the current status of the proton tensor charge, obtained as the first Mellin moment of the chiral-odd transversity parton distribution extracted from a global analysis of data for inclusive pion-pair production in deep-inelastic scattering and in proton-proton collisions with one transversely polarized proton. We compare this result with other phenomenological extractions and with the direct computation on lattice of the tensor charge as hadronic matrix element of the local chiral-odd tensor operator. The knowledge of the proton tensor charge is important among other things for detecting possible signals of new physics in high-precision low-energy experiments.

XXVII International Workshop on Deep-Inelastic Scattering and Related Subjects - DIS2019

8-12 April, 2019

Torino, Italy

${ }^{*}$ Speaker. 


\section{Introduction}

The transversely polarized parton distribution $h_{1}$ (transversity) is interesting because its first Mellin moment, the so-called tensor charge, can be useful to search for effects from physics beyond the Standard Model (BSM) [1]. On the other hand, the $h_{1}$ is a chiral-odd object and can be extracted only from processes with at least two hadrons: either semi-inclusive deep-inelastic scattering (SIDIS) or hadronic collisions.

Here, we address the transversity in the semi-inclusive production of unpolarized hadron pairs with small invariant mass [2]. For each process above, the leading-twist cross section contains a specific modulation in the azimuthal angles of the final-state products whose coefficient is given by the same universal simple product $h_{1} H_{1}^{\varangle}$, where $H_{1}^{\varangle}$ is a chiral-odd di-hadron fragmentation function (DiFF) quantifying the correlation between the transverse polarization of the fragmenting quark and the relative momentum of the detected hadron pair [3, 4, 5, 6, 7, 8, 9]. The same polarized DiFF can be independently extracted by looking at correlations between the azimuthal orientations of two hadron pairs in back-to-back jets in $e^{+} e^{-}$annihilations [10, 11, 12, 13].

Experimental data for the inclusive $\left(\pi^{+} \pi^{-}\right)$pair production in SIDIS were collected by the HERMES collaboration for a proton target [14], and by the COMPASS collaboration for both protons and deuterons $[15,16]$. The function $H_{1}^{\varangle}$ was first extracted [12] from data on the distribution of back-to-back $\left(\pi^{+} \pi^{-}\right)$pairs in $e^{+} e^{-}$annihilations measured by the BELLE collaboration [17]. In Ref. [18], the STAR collaboration published the first results for the azimuthal distribution of $\left(\pi^{+} \pi^{-}\right)$pairs produced in proton-proton collisions with a transversely polarized proton, predicted long before in Ref. [8]. The final extraction of the transversity $h_{1}$ from a global fit of all these data has been detailed in Ref. [19].

In the following, we discuss the first Mellin moment of $h_{1}$ and we compare it with the results from other phenomenological extractions and from the direct computation of the proton tensor charge on the lattice.

\section{The global fit}

Our global fit is based on a functional form for $h_{1}$ that satisfies the Soffer bound at any value of the parton fractional momentum $x$ and at any hard scale $Q$ of the process [6,7]. Moreover, we require that the Mellin transform of $h_{1}$ can be analytically computed in order to speed up the execution of that part of the code dealing with the proton-proton collision matrix element [19]. For these reasons, at the starting scale $Q_{0}^{2}=1 \mathrm{GeV}^{2}$ the valence component $q_{v}$ of the transversity $h_{1}$ has the following form:

$$
\begin{aligned}
x h_{1}^{q_{v}}\left(x, Q_{0}^{2}\right) & =F^{q}(x) F_{\mathrm{SB}}^{q}(x), \\
F_{\mathrm{SB}}^{q}(x) & =N_{\mathrm{SB}}^{q} x^{a_{q}}(1-x)^{b_{q}}\left(1+c_{q} \sqrt{x}+d_{q} x+e_{q} x^{2}+f_{q} x^{3}\right), \\
F^{q}(x) & =N_{F}^{q} \frac{\mathscr{F}^{q}(x)}{\max _{x}\left[\left|\mathscr{F}^{q}(x)\right|\right]}, \quad \mathscr{F}^{q}(x)=x^{A_{q}}\left[1+B_{q} T_{1}(x)+C_{q} T_{2}(x)+D_{q} T_{3}(x)\right] .
\end{aligned}
$$

The $F_{\mathrm{SB}}$ in Eq. (2.2) is a fit to the Soffer bound at $Q_{0}^{2}$, whose analytic expression is listed in the appendix of Ref. [6]. Using the parameters listed in Tab.I of Ref. [19], the accuracy is of order $1 \%$ in the range $0.001 \leq x \leq 1$. In Eq. (2.3), the $T_{n}(x)$ are the Cebyshev polynomials of order 
$n$. The $N_{F}^{q}, A_{q}, B_{q}, C_{q}, D_{q}$, are the fitting parameters. In fixed-target SIDIS experiments, only the valence components of transversity can be accessed [20,6]; hence, we have in total 10 free parameters. If we impose the constraint $\left|N_{F}^{q}\right| \leq 1$, then $\left|F^{q}(x)\right| \leq 1$ for all $x$, and the $h_{1}^{q_{v}}$ in Eq. (2.1) automatically satisfies the Soffer inequality at any scale. Another constraint comes from the low- $x$ behavior of transversity, which from the above equations looks like $x h_{1}^{q_{v}}(x) \approx x^{A_{q}+a_{q}}$. For the given $a_{q}$, the parameter $A_{q}$ is strongly constrained by requiring that the tensor charge $\delta q\left(Q^{2}\right)$ is finite. We numerically evaluate the Mellin moment in the range $\left[10^{-6}, 1\right]$. In order to avoid uncontrolled extrapolation errors at $0 \leq x<10^{-6}$, we impose the condition $A_{q}+a_{q}>1 / 3$, which also grants that $\delta q$ is evaluated at $1 \%$ accuracy.

The statistical uncertainty of the global fit is studied at the $90 \%$ confidence level using the same bootstrap method as in our previous fits [6,7]. In the analysis of di-hadron $e^{+} e^{-}$data, the unconstrained gluon channel is assumed $D_{1}^{g}\left(Q_{0}^{2}\right)=0$. We parametrize this error by computing the asymmetry also with $\left.D_{1}^{g} Q_{0}^{2}\right)=D_{1}^{u}\left(Q_{0}^{2}\right) / 4$, or $D_{1}^{u}\left(Q_{0}^{2}\right)$. We have verified that these choices alter the $\chi^{2}$ of the $e^{+} e^{-}$fit in Ref. [12] by $10-50 \%$, keeping always $\chi^{2} /$ dof $\lesssim 2$. The number of replicas is fixed by reproducing the mean and standard deviation of the original data points. For each option, it turns out that 200 replicas are sufficient. Hence, we have in total 600 replicas.
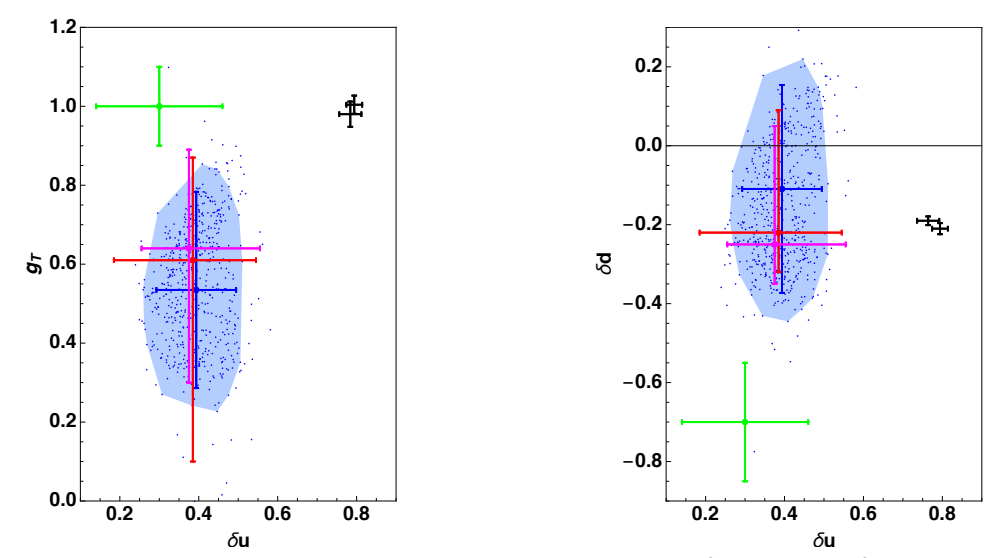

Figure 1: Left panel: isovector $g_{T}$ vs. up $\delta u$ tensor charges at $Q^{2}=4 \mathrm{GeV}^{2}$ and $90 \%$ confidence level. Blue cross and points with shaded area for this global fit [19], red cross from Ref. [21], magenta cross from Ref. [22], green cross from Ref. [23], black crosses for lattice results from Ref. [24] (lower cross) and [25] (higher cross), respectively. Right panel: same for $\delta d$ vs. $\delta u$ (position of two lattice crosses interchanged).

\section{Results}

In Fig. 1, we show the results for the tensor charge $\delta q$ at $Q^{2}=4 \mathrm{GeV}^{2}$ and $90 \%$ confidence level. The left panel plots the isovector component $g_{T} \equiv \delta u-\delta d$ vs. the up $\delta u$, the right panel the down component $\delta d$ vs. $\delta u$. In all panels, the blue cross is the result of our global fit [19]. Each blue single point is the result of a replica and the blue shadow encompasses the area of the $90 \%$ confidence level. The red cross is the result of the phenomenological extraction based on the Collins effect in the transverse-momentum dependent framework [21], the magenta cross is also based on the Collins effect but in an extended parton model framework [22], the green cross is the result obtained with a nested Monte Carlo iterative approach to the Collins effect but with the 
additional constraint to reproduce the average lattice result for $g_{T}$ [23]. Finally, the black crosses are the latest lattice results: in the left panel, the lower cross is from the PNDME collaboration [24] while the higher cross from the ETMC one [25]; in the right panel, the order is reversed.

We observe that within statistical errors all the phenomenological extractions of transversity produce the same components of tensor charge, they are in agreement with lattice results for $\delta d$ (although within very large error bars, see right panel) but they disagree on $\delta u$ and, consequently, on $g_{T}$ by more than $2 \sigma$ (see left panel). The phenomenological analysis of Ref. [23] is peculiar because it is the only one including the constraint to reproduce the lattice result for $g_{T}$ (see left panel). However, this comes at the price of getting a large discrepancy with lattice not only in $\delta u$ but also in the $\delta d$ component, as shown by the green cross in the right panel. This suggests that the enforced compatibility on $g_{T}=\delta u-\delta d$ might result from the artificial balance of two anomalous results for $\delta u$ and $\delta d$. We cross-checked this hypothesis by repeating also our global fit with the constraint to reproduce the lattice result for $g_{T}$ : similarly to Ref. [23], the fit converges at the price of deteriorating the previous agreement with lattice for the $\delta d$ component; also the statistical quality of the fit slightly deteriorates from a $\chi^{2} / \mathrm{dof}=1.76 \pm 0.11$ to $\chi^{2} / \mathrm{dof}=1.82 \pm 0.25$.
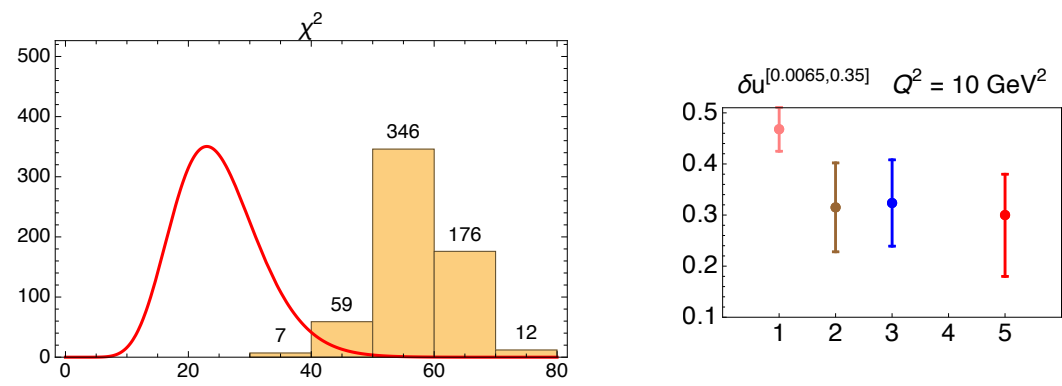

Figure 2: Left panel: probability density function for the $\chi^{2}$ distribution of 600 replicas from our global fit reproducing also the average lattice results for all components of tensor charge. Right panel: the truncated up tensor charge at $Q^{2}=10 \mathrm{GeV}^{2}$. Red point (label 5) from Ref. [21], blue point (label 3) from our global fit of Ref. [19], brown point (label 2) by constraining our fit to reproduce the lattice $g_{T}$, pink point (label 1) by further constraining it to reproduce also the lattice $\delta u$ and $\delta d$.

We explored also the option of constraining our global fit to reproduce the average lattice result for all the components of the tensor charge. In Fig. 2, we show in the left panel the probability density function for the corresponding distribution of the $\chi^{2}$ obtained from each of the 600 replicas. The red line corresponds to the ideal situation of a $\chi^{2} / \mathrm{dof}=1$. The histogram of the resulting 600 replicas is instead markedly shifted to the right and its overlap with the ideal situation is negligible. In fact, the overall statistical quality of the fit further deteriorates with respect to the previous cases, resulting in a global $\chi^{2} /$ dof $=2.29 \pm 0.25$. In the right panel, we show the truncated up tensor charge $\delta u^{[0.0065,0.35]}$, namely the truncated Mellin moment of $h_{1}^{u_{v}}$ when the calculation of the integral is limited to the $x$-range $[0.0065,0.35]$ covered by the experimental points. At $Q^{2}=10$ $\mathrm{GeV}^{2}$, there is almost a perfect match between the result from the phenomenological extraction of Ref. [21] (red point with label 5) and our global fit in Ref. [19] (blue point with label 3). Adding to our global fit the constraint to reproduce the lattice $g_{T}$ does not alter much the situation (see the brown point with label 2). However, if we further require to reproduce also the $\delta u$ and $\delta d$ from lattice, the value of $\delta u^{[0.0065,0.35]}$ sharply changes to the pink point with label 1 . The instability of the truncated Mellin integral, computed in the $x$-range where experimental data can effectively 
constrain the $x$-dependence of the transversity, is very suspicious; even more so if this latter result for $\delta u^{[0.0065,0.35]}$ (the pink point) corresponds to a statistically disfavoured solution.

\section{Conclusion}

We discussed the current status of the proton tensor charge, obtained as the first Mellin moment of the chiral-odd transversity parton distribution extracted from a global analysis of data for inclusive pion-pair production in deep-inelastic scattering and in proton-proton collisions with one transversely polarized proton. We compared our result with other phenomenological extractions and with the direct computation on lattice of the tensor charge as hadronic matrix element of the local chiral-odd tensor operator.

We deduce that currently there is no simultaneous compatibility between the results of lattice and phenomenology for all the components of the proton tensor charge, namely up $(\delta u)$, down $(\delta d)$ and isovector $\left(g_{T}\right)$ ones. On the other hand, the knowledge of the proton tensor charge is important among other things for detecting possible signals of new physics in high-precision low-energy experiments. Hence, on one side more work is needed to scrutinize the lattice computation, while on the other side more effort and data are needed to improve the precision of the phenomenological extraction of transversity.

\section{Acknowledgments}

Most of the results presented in this report have been carried out in collaboration with A. Bacchetta, to whom I am deeply indebted. This research is partially supported by the European Research Council (ERC) under the European Union's Horizon 2020 research and innovation program (Grant Agreement No. 647981, 3DSPIN).

\section{References}

[1] A. Courtoy, S. Baessler, M. Gonzalez-Alonso, and S. Liuti, Beyond-Standard-Model Tensor Interaction and Hadron Phenomenology, Phys. Rev. Lett. 115 (2015) 162001, [arXiv:1503.0681].

[2] J. C. Collins and G. A. Ladinsky, On $\pi-\pi$ correlations in polarized quark fragmentation using the linear sigma model, http://arXiv.org/abs/hep-ph/9411444.

[3] M. Radici, R. Jakob, and A. Bianconi, Accessing transversity with interference fragmentation functions, Phys. Rev. D65 (2002) 074031, [http: / / arXiv.org/abs/hep-ph/0110252].

[4] A. Bacchetta and M. Radici, Partial-wave analysis of two-hadron fragmentation functions, Phys. Rev. D67 (2003) 094002, [hep-ph / 0212300$].$

[5] A. Bacchetta, A. Courtoy, and M. Radici, First glances at the transversity parton distribution through dihadron fragmentation functions, Phys.Rev.Lett. 107 (2011) 012001, [arXiv:1104.3855].

[6] A. Bacchetta, A. Courtoy, and M. Radici, First extraction of valence transversities in a collinear framework, JHEP 1303 (2013) 119, [arXiv: 1212.3568].

[7] M. Radici, A. Courtoy, A. Bacchetta, and M. Guagnelli, Improved extraction of valence transversity distributions from inclusive dihadron production, JHEP 05 (2015) 123, [arXiv: 1503.0349 ].

[8] A. Bacchetta and M. Radici, Dihadron interference fragmentation functions in proton- proton collisions, Phys. Rev. D70 (2004) 094032, [hep-ph / 0409174 ]. 
[9] M. Radici, A. M. Ricci, A. Bacchetta, and A. Mukherjee, Exploring universality of transversity in proton-proton collisions, Phys. Rev. D94 (2016), no. 3 034012, [arXiv: 1604 . 0658 ].

[10] D. Boer, R. Jakob, and M. Radici, Interference fragmentation functions in electron positron annihilation, Phys. Rev. D67 (2003) 094003, [hep-ph/ 0302232 ].

[11] A. Bacchetta, F. A. Ceccopieri, A. Mukherjee, and M. Radici, Asymmetries involving dihadron fragmentation functions: from DIS to e+e-annihilation, Phys. Rev. D79 (2009) 034029, [arXiv:0812.0611].

[12] A. Courtoy, A. Bacchetta, M. Radici, and A. Bianconi, First extraction of Interference Fragmentation Functions from $e^{+} e^{-}$data, Phys.Rev. D85 (2012) 114023, [arXiv:1202.0323].

[13] H. H. Matevosyan, A. Bacchetta, D. Boer, A. Courtoy, A. Kotzinian, M. Radici, and A. W. Thomas, Semi-inclusive production of two back-to-back hadron pairs in $e^{+} e^{-}$annihilation revisited, Phys. Rev. D97 (2018), no. 7 074019, [arXiv:1802.0157].

[14] HERMES Collaboration, A. Airapetian et al., Evidence for a Transverse Single-Spin Asymmetry in Leptoproduction of pi+pi-Pairs, JHEP 06 (2008) 017, [arXiv: 0803.2367 ].

[15] COMPASS Collaboration, C. Adolph et al., Transverse spin effects in hadron-pair production from semi-inclusive deep inelastic scattering, Phys.Lett. B713 (2012) 10-16, [arXiv: 1202 . 6150].

[16] COMPASS Collaboration, C. Adolph et al., A high-statistics measurement of transverse spin effects in dihadron production from muon-proton semi-inclusive deep-inelastic scattering, Phys.Lett. $\mathbf{B 7 3 6}$ (2014) 124-131, [arXiv:1401.7873].

[17] Belle Collaboration Collaboration, A. Vossen et al., Observation of transverse polarization asymmetries of charged pion pairs in e+e-annihilation near sqrt s=10.58 GeV, Phys.Rev.Lett. 107 (2011) 072004, [arXiv:1104.2425].

[18] STAR Collaboration, L. Adamczyk et al., Observation of Transverse Spin-Dependent Azimuthal Correlations of Charged Pion Pairs in $p^{\uparrow}+p$ at $\sqrt{s}=200$ GeV, Phys. Rev. Lett. 115 (2015) 242501, [arXiv:1504.0041].

[19] M. Radici and A. Bacchetta, First Extraction of Transversity from a Global Analysis of Electron-Proton and Proton-Proton Data, Phys. Rev. Lett. 120 (2018), no. 19192001, [arXiv:1802.0521].

[20] A. Bacchetta and M. Radici, Modeling dihadron fragmentation functions, Phys. Rev. D74 (2006) 114007, [hep-ph/0608037].

[21] Z.-B. Kang, A. Prokudin, P. Sun, and F. Yuan, Extraction of Quark Transversity Distribution and Collins Fragmentation Functions with QCD Evolution, Phys. Rev. D93 (2016), no. 1014009 , [arXiv:1505.0558].

[22] M. Anselmino, M. Boglione, U. D’Alesio, S. Melis, F. Murgia, et al., Simultaneous extraction of transversity and Collins functions from new SIDIS and e+e-data, Phys.Rev. D87 (2013) 094019 , [arXiv:1303.3822].

[23] H.-W. Lin, W. Melnitchouk, A. Prokudin, N. Sato, and H. Shows, First Monte Carlo global analysis of nucleon transversity with lattice QCD constraints, arXiv:1710.0985.

[24] T. Bhattacharya, V. Cirigliano, S. Cohen, R. Gupta, H.-W. Lin, and B. Yoon, Axial, Scalar and Tensor Charges of the Nucleon from 2+1+1-flavor Lattice QCD, Phys. Rev. D94 (2016), no. 5054508 , [arXiv:1606.0704].

[25] C. Alexandrou et al., Nucleon scalar and tensor charges using lattice QCD simulations at the physical value of the pion mass, Phys. Rev. D95 (2017), no. 11 114514, [arXiv: 1703.0878 ]. 